Psychother Psychosom 2016;85:114-116

DOI: $10.1159 / 000441458$

\title{
Critical Slowing Down as a Personalized Early Warning Signal for Depression
}

\author{
Marieke Wichers ${ }^{\mathrm{a}, \mathrm{c}}$, Peter C. Groot ${ }^{\mathrm{b}}$, Psychosystems, ESM Group, \\ EWS Group \\ ${ }^{a}$ Department of Psychiatry and Psychology, School for \\ Mental Health and Neuroscience, Maastricht University, and \\ ${ }^{b}$ Department of Psychiatry and Psychology, User Research \\ Centre, Maastricht University Medical Centre, Maastricht, \\ and 'Interdisciplinary Center Psychopathology and Emotion \\ Regulation, University of Groningen, University Medical Centre \\ of Groningen, Groningen, The Netherlands
}

The etiology of mental disorders such as major depression (MD) involves continuously ongoing gene-environment interactions that act in highly person-specific ways. This hampers the accurate assessment of personalized risk and likelihood for transitions in symptomatology. Complex dynamical system theory may provide a new route to assessment of personalized risk for transitions on the continuum of depression (of which MD forms the extreme end). In complex systems, it has been demonstrated that generic early warning signals, signaling critical slowing down (CSD) of the system, may often precede critical transitions [1]. It has been hypothesized previously that depression also behaves as a complex dynamical system as both sudden rises in depressive symptoms and sudden gains (disappearance of symptoms) are frequent phenomena. This suggests that fragility of the system builds up gradually, under the surface, and eventually reaches the 'tipping point' to result in a sudden critical transition. This is a typical characteristic of complex dynamical systems. This phenomenon of rising fragility of the system is reflected in CSD, which occurs when the system requires increased periods of time to recover from small perturbations [1]. Statistically, CSD can be detected through increased temporal autocorrelation and variance within elements of the system and increased spatial correlation (network connections) between those elements. These are the indicators that we refer to as early warning signals (EWS) [1,2]. A recent study was the first to explicitly examine whether these generic EWS also occur in depression. This study involved two separate samples (one general population, $\mathrm{n}=535$, and one depressed sample, $\mathrm{n}=93$ ) [3]. In both samples, higher levels of all three hypothesized EWS at baseline were found in individuals close to a future change in symptoms (based on follow-up measurements of depressive symptoms) compared to individuals who were not experiencing a change in symptoms in the near future [3]. This study, however, did not yet directly observe rising EWS patterns within individuals

\section{KARGER}

E-Mail karger@karger.com www.karger.com/pps

\section{The Author(s) \\ Published by S. Karger AG, Basel 0033-3190/16/0852-0114\$39.50/0}

This article is licensed under the Creative Commons AttributionNonCommercial-NoDerivatives 4.0 International License (CC BY NC-ND) (http://www.karger.com/Services/OpenAccessLicense). Usage and distribution for commercial purposes as well as any distribution of modified material requires written permission. that anticipated these individuals' critical transitions in depressive symptoms. Such direct evidence is still missing. Only if we find that, within single individuals, EWS temporally and systematically anticipate observed qualitative shifts in mood, then these ideas may transform into precise and person-specific information on risk for transitions in depression [4]. To date, no study has yet succeeded in gathering the required observations to obtain such direct evidence: time series of experiential states that cover a demonstrable critical transition, as well as a sufficient number of observations in advance of that transition to allow for prospective measures of EWS.

This case experiment presents precisely such data. It was conducted by a mental health care user (hereafter: the participant) who collected 1,474 prospective momentary observations of daily life experiences. The participant is male, aged 57 years, has a history of multiple episodes of MD and has been using antidepressants the previous 8.5 years (for details, see Groot [5]). The participant monitored - using a semi-random experience-sampling protocol - his momentary experiential states over the course of gradual discontinuation (from 150 to $0 \mathrm{mg}$ over 8 weeks) of his antidepressant (venlafaxine). The participant and researchers were blind to the dose reduction scheme that was chosen from various schemes, which varied in the time (0-6 weeks) before dose reduction would start (fig. 1a). The participant collected reports of momentary states up to 10 times a day over a period of 239 days. Affect items used were irritated, content, lonely, anxious, enthusiastic, cheerful, guilty, indecisive, strong, restless and agitated. These yielded three principal components: negative affect, positive affect and mental unrest. In addition, subclinical psychotic experiences were measured with the item 'suspicious'. The cognitive context was measured with the item 'worrying'. All momentary states were rated on a 7 -point scale. Time trends (slow nonlinear alterations in mean levels) were removed before analysis. Further details on the statistical techniques used are available upon request (including data set and R scripts).

Figure 1a (second panel) shows that a sudden shift in depressive symptoms was observed around day 127 of the experiment. Change point analysis confirmed the presence of a statistically significant transition ( $p=0.005)$. The shift was also clinically relevant, as the participant and his psychiatrist decided to resume the use of antidepressants 36 days after the studied period.

This transition in symptoms was preceded by CSD. Figure 1a (lower panels) shows patterns of rising temporal autocorrelations

The following people belong to the research consortia that contributed to this paper: Psychosystems: D. Borsboom, A.O.J. Cramer, S. Epskamp, K.S. Kendler, H.L.J. van der Maas, F. Tuerlinckx and J.T.W. Wigman; ESM Group: P. Delespaul, F. Peeters, C.J.P. Simons and E. Snippe; EWS Group: I.A. van de Leemput and M. Scheffer.
Prof. Dr. M. Wichers, Interdisciplinary Center Psychopathology and Emotion Regulation, University Medical Centre of Groningen University of Groningen, Triade Building entrance 24, internal code CC72 PO Box 30.001, NL-9700 RB Groningen (The Netherlands) E-Mail m.c.wichers@ umcg.nl 
Fig. 1. a The time line of the experiment. The upper panel depicts the experimental design, the second panel shows the raw weekly measurements of depressive symptoms using the SCL-90-R depression subscale. The third and fourth panels show the moving window (width $=30$ days) in autocorrelation and variance results, respectively, based on the detrended overall mental state variable (total mental state score based on a moving window over time). Separate analyses were performed on the detrended variables to obtain the autocorrelation and variance for each data window. The gray vertical bar reflects the time window where the transition in depressive symptoms took place. $\mathbf{b}$ The change in the network structure (reflecting spatial correlation) over the course of the experiment using data only obtained before day 128 (the moment of the transition in depressive symptoms) between the five mental state components measured. Significant connections and their strength (reflected in the $\beta$-coefficient) are shown. The dashed arrows indicate negative and the continuous arrows positive time-lagged effects. $\mathrm{AD}=$ Antidepressant; $\mathrm{PA}=$ positive affect; $\mathrm{MU}=$ mental unrest; $\mathrm{NA}=$ negative affect; $\mathrm{WO}=$ worry; SU = suspicious.

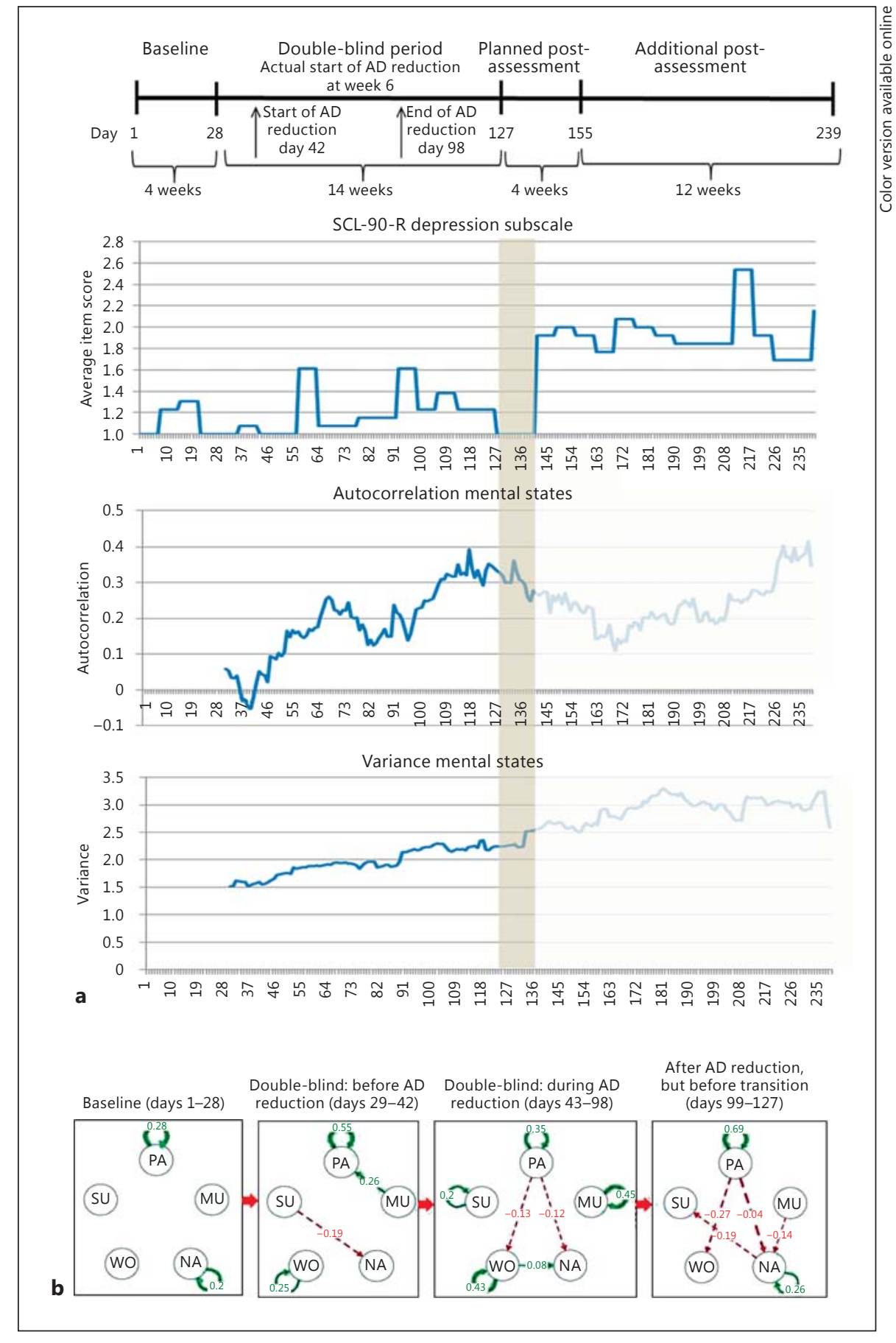

and variance - based on the summed detrended score of the five mental states - in the period before the critical transition. It seems unlikely that autocorrelation and variance are a consequence of floor effects, as time trends were removed in the autocorrelation analyses, autocorrelations showed different dynamics over time compared to variance, and effects were robustly present in variables that were far removed from the end of the response scale during the entire time series.

Personalized Early Warning Signals in Depression
Furthermore, networks of dynamic relations between mental states were analyzed (spatial correlations) [6]. As the shift in mood approached, the estimated structure changed to include both more and stronger connections between the mental states (fig. 1b). Continuous changes in the dynamic network structure of the affective states are visualized in a movie that tracks the evolution of the network over time (https://www.dropbox.com/s/nppjlnpu8a7k6n8/ DepressionTheMovie10\%20\%281\%29.avi?dl=0). 
Our findings further support the hypothesis that depression can be characterized as a complex dynamical system. This is the first study showing rising EWS within a single person anticipating a clinically and statistically significant transition in depression.

Furthermore, these results bolster a growing body of work arguing that depression can be understood as a complex network of mental states that trigger each other [7-9]. In case of high network connectivity, activation of a single node (mental state) may initiate a cascade of effects that keeps resonating in the network.

The generic principles of complex dynamical systems may offer new possibilities for anticipating person-specific shifts in depression, even if we cannot yet fully grasp the mechanistic details of the condition. Furthermore, the current intensive monitoring approach closely aligns with existing recommendations for detecting prodromal phases of MD [10]. In short, the current approach may offer a new way in the field of psychiatry to acquire personalized and clinically relevant information.

\section{Acknowledgments}

This work was supported by an Aspasia grant (to M.W., NWO grant), by the Brain Foundation of the Netherlands [to M.W., grant No. F2012(1)-03] and by a VICI grant (to P. de Jonge, NWO grant No. 91812607).

Disclosure Statement

There is no conflict of interest.

\section{References}

1 Scheffer M, Bascompte J, Brock WA, Brovkin V, Carpenter SR, Dakos V, et al: Early-warning signals for critical transitions. Nature 2009;461:5359 .

2 Dakos V, Van Nes EH, Donangelo R, Fort H, Scheffer M: Spatial correlation as leading indicator of catastrophic shifts. Theor Ecol 2010;3:163.

- 3 Van de Leemput IA, Wichers M, Cramer AO, Borsboom D, Tuerlinckx F, Kuppens P, et al: Critical slowing down as early warning for the onset and termination of depression. Proc Natl Acad Sci USA 2014;111:87-92.

4 Bos EH, De Jonge P: 'Critical slowing down in depression' is a great idea that still needs empirical proof. Proc Natl Acad Sci USA 2014;111:E878.

5 Groot PC: Patients can diagnose too: how continuous self-assessment aids diagnosis of, and recovery from, depression. J Ment Health 2010;19: 352-362.

6 Bringmann LF, Vissers N, Wichers M, Geschwind N, Kuppens P, Peeters F, et al: A network approach to psychopathology: new insights into clinical longitudinal data. PLoS One 2013;8:e60188.

7 Borsboom D, Cramer AO: Network analysis: an integrative approach to the structure of psychopathology. Annu Rev Clin Psychol 2013;9:91121.

8 Wichers M: The dynamic nature of depression: a new micro-level perspective of mental disorder that meets current challenges. Psychol Med 2014;44:1349-1360.

9 Kendler KS, Zachar P, Craver C: What kinds of things are psychiatric disorders? Psychol Med 2011;41:1143-1150.

10 Fava GA, Tossani E: Prodromal stage of major depression. Early Interv Psychiatry 2007;1:9-18. 